\title{
Isotermas de dessorção de resíduos de abacaxi
}

\author{
H. V. ALEXANDRE ${ }^{1}$, F. L. H. da SILVA ${ }^{2}$, J. P. GOMES ${ }^{3}$, O. S. da SILVA ${ }^{4}$ e JOÃO P. \\ CARVALHO
}

\author{
${ }^{1}$ Doutora em Engenharia de Processos \\ ${ }^{2}$ Universidade Federal da Paraíba, Departamento de Química \\ ${ }^{3}$ Universidade Federal de Campina Grande, Unidade Acadêmica em Engenharia Agrícola \\ ${ }^{4}$ Universidade Federal de Campina Grande, Unidade Acadêmica de Tecnologia de Alimentos \\ ${ }^{5}$ Aluno de Pós-Graduação em Engenharia Química, Universidade Federal de Campina Grande \\ E-mail para contato: hofsky@gmail.com
}

RESUMO - Resíduos sólidos de abacaxi (casca e coroa) têm obtido interesse em pesquisa devido ao considerável volume produzido e presença de substâncias com grande variedade de aplicações. A elevada umidade do resíduo, principalmente a casca, torna necessária a sua secagem antes de um possível aproveitamento, no entanto, a qualidade e a vida útil dos pós dependem de seus teores de água e da maneira como esta se encontra ligada ao alimento. $\mathrm{O}$ comportamento higroscópico de alimentos em pó pode ser avaliado por meio de suas isotermas de sorção. Objetivou-se com esse trabalho, determinar as isotermas de dessorção de resíduos do abacaxi e ajustar modelos matemáticos aos dados experimentais obtidos pelo método gravimétrico estático e verificar a possibilidade de utilização de um único modelo. $\mathrm{O}$ intervalo de teor de umidade estudado variou entre 0,083 e 1,265 g de $\mathrm{H}_{2} \mathrm{O} / \mathrm{g}$ de matéria seca e 0,079 e 1,79 $0 \mathrm{H}_{2} \mathrm{O} / \mathrm{g}$ de matéria seca, para casca e coroa, respectivamente, enquanto a atividade de água variou entre 0,079 e 0,914 para casca e 0,106 e 0,921 para coroa. Aos dados experimentais foi avaliado o ajuste dos modelos de GAB e BET linearizado, por meio do coeficiente de determinação e do desvio médio relativo. Segundo os parâmetros estatísticos de análise, o modelo de GAB foi o que descreveu melhor a higroscopicidade dos resíduos do abacaxi.

\section{INTRODUÇÃO}

O Brasil é um dos maiores produtores de abacaxi ressaltando- se, como os principais, os estados da Paraíba, Pará e Minas Gerais. Brito Neto et al. (2008) afirmam que as condições brasileiras para a produção deste fruto visando aos mercados interno e externo, lhes asseguram vantagens comparativas em relação aos países concorrentes devido ao clima favorável, grande disponibilidade de área e de tecnologias.

O abacaxi é utilizado tanto para o consumo in natura quanto na industrialização, em diferentes formas: pedaços em calda, suco, pedaços cristalizados, geleias, licor, vinho, vinagre e aguardente. Como subproduto desse processo industrial pode-se obter ainda: álcool, ácidos cítrico, málico e ascórbico e rações para animais e com isso cresce a geração de resíduo que será disponibilizado pela agroindústria processadora do fruto.

O resíduo industrial, depois de gerado, necessita de destino adequado, pois não pode ser acumulado indefinidamente no local em que foi produzido. A crescente preocupação com o 
meio ambiente incentiva a viabilização de projetos que levam à sustentabilidade do sistema de produção industrial. A indústria de alimentos produz uma série de resíduos com alta capacidade de reutilização. Com isto, minimiza-se o impacto ambiental desses tipos de indústria na região em que estão situadas e ainda se agrega valor aos produtos do mercado (Pelizer et al., 2007).

No entanto, o que limita a utilização destes resíduos é o seu alto teor de umidade. Sendo assim, a secagem representa uma alternativa para a preservação e aproveitamento alimentício da casca e coroa da indústria processadora de abacaxi.

De acordo com Heldman \& Hartel (2000) quando um produto alimentício é submetido a secagem tanto seu teor de umidade como a atividade de água se alteram ao longo do processo devido à relação entre o teor de umidade de equilíbrio do produto e a umidade relativa do ar. Portanto, o estudo desses parâmetros é de grande importância para o desenvolvimento de um processo de secagem haja vista que especifica o teor de umidade do alimento, que pode ser atingido sob condições de ar de secagem específico.

O conhecimento das isotermas de sorção de umidade dos alimentos tem aplicação na predição do tempo de secagem, vida útil do produto, determinação do tipo de embalagem e na caracterização do produto, inclusive quando o mesmo é constituído por componentes de atividade de água diferentes (Alexandre et al., 2007). Elas são obtidas a partir de dados de adsorção (ganho de umidade) e dessorção (perda de umidade), respectivamente; mas podem ser preditas através de modelos matemáticos classicamente utilizados com essa finalidade, amplamente encontrados na literatura (Akanbi et al., 2006; Assunção \& Pena, 2007; Furmaniak et al., 2007).

Vários pesquisadores têm estudado o comportamento higroscópico de diversos produtos agrícolas que descrevem modelos matemáticos distintos para expressar o teor de água de equilíbrio em função da temperatura e umidade relativa do ar (Sousa et al., 2013).

Ante a importância do conhecimento da higroscopicidade dos produtos agrícolas objetivou-se, neste trabalho, determinar as isotermas de dessorção dos resíduos de abacaxi (casca e coroa) para diversas condições de temperatura e atividades de água e ajustar diferentes modelos matemáticos aos dados experimentais selecionando-se aquele que melhor representa o fenômeno.

\section{MATERIAIS E MÉTODOS}

A matéria-prima utilizada no estudo foi o resíduo (casca e coroa) do abacaxi pérola (Ananas comosus L.); o fruto foi adquirido na EMPASA na cidade de Campina Grande, PB. No laboratório o resíduo (casca e coroa) foi separado da polpa em seguida acondicionado em sacos de polietileno e só então armazenado em freezer a $-22{ }^{\circ} \mathrm{C}$ até o momento de ser utilizado nos experimentos. 
Teor de umidade: Foi determinado conforme descrito por BRASIL (2005) em estufa a $105^{\circ} \mathrm{C}$ até peso constante.

Atividade de água: Para o levantamento dos dados das isotermas de dessorção do resíduo do abacaxi foi utilizado o equipamento Thermoconstanter Novasina TH200. Este equipamento é projetado para a medida de atividade de água $\left(\mathrm{a}_{\mathrm{w}}\right)$ com a temperatura controlada. No aparelho um sensor mede a atividade de água à temperatura indicada de pequenas amostras e o transmissor RTD-20 converte os sinais da célula de medição, indicando os valores medidos de $\mathrm{a}_{\mathrm{w}} \mathrm{e}$ de temperatura diretamente no mostrador.

Isotermas de dessorção: As isotermas de dessorção foram determinadas por meio do método estático indireto. Para isso, 2,0 $\mathrm{g}$ de amostra foram pesados em recipiente apropriado e secos por diferentes períodos em estufa com circulação forçada de ar, a $55^{\circ} \mathrm{C}$, de modo a obter valores de teor de umidade e de atividade de água dispostos em todo o intervalo estudado. Após cada período de secagem foi determinada a atividade de água, em triplicata , utilizando o equipamento Thermoconstanter Novasina TH200, nas temperaturas de $25,30,35$ e $40^{\circ} \mathrm{C}$ e também realizada a pesagem da amostra para a obtenção da massa no equilíbrio $\left(\mathrm{m}_{\mathrm{eq}}\right)$ utilizada na determinação do teor de umidade de equilíbrio. Esse procedimento foi repetido até que a última atividade de água lida fosse igual ou superior à anterior. A sucessão dessas leituras indica os pontos da curva da isoterma de dessorção dos resíduos do abacaxi.

Umidade de equilíbrio: Posteriormente à determinação desses pontos, as amostras foram levadas à estufa sem circulação de ar, a $105^{\circ} \mathrm{C}$ por $24 \mathrm{~h}$ para determinação da massa seca $\left(\mathrm{m}_{\mathrm{s}}\right)$ e posteriormente a umidade de equilíbrio (Equação 1).

$$
X_{e q}=\frac{m_{e q}-m_{S}}{m_{S}}
$$

Para o ajuste matemático dos dados experimentais utilizou-se modelos matemáticos, e fez-se a correspondência entre a umidade do produto em questão $\left(X_{\text {eq }}\right)$ e a atividade de água $\left(\mathrm{a}_{\mathrm{w}}\right)$ fornecendo importantes informações sobre o fenômeno de dessorção. Para o ajuste das isotermas de dessorção da casca e coroa do abacaxi a análise de regressão foi realizada com auxílio do aplicativo Statistica 5.0, utilizando a metodologia de estimativa de Quasi-Newton para o modelo de GAB (Equação 2). Para o modelo de BET linearizado (Equação 3), foi utilizada a metodologia de estimativa Simplex.

$$
\frac{X_{e}}{X_{m}}=\frac{\left(C_{G A B}-1\right) K_{G A B} a_{w}}{1+\left(C_{G A B}-1\right) K_{G A B} a_{w}}+\frac{K_{G A B}}{1-K_{G A B} a_{w}}
$$




$$
\frac{a_{w}}{\left(1-a_{w}\right) X_{e}}=\frac{1}{X_{m} C_{B E T}}+\frac{a_{w}\left(C_{B E T}-1\right)}{X_{m} C_{B E T}}
$$

Os critérios usados para determinação do melhor ajuste dos modelos aos dados experimentais foram: o coeficiente de determinação $\left(\mathrm{R}^{2}\right)$ e o desvio percentual médio $(\mathrm{P})$, calculado pela Equação 4.

$$
P=\frac{100}{n} \cdot \sum_{i=1}^{n} \frac{\mid\left(X_{e q_{(\exp )}}-X_{\left.e q_{(t e o r)}\right)}\right)}{X_{e q_{(\exp )}}}
$$

\section{RESULTADOS E DISCUSSÃO}

$\mathrm{O}$ resíduo de abacaxi apresentou um teor de umidade médio de 1,208 g de $\mathrm{H}_{2} \mathrm{O} / \mathrm{g}$ para casca e 1,291 g de $\mathrm{H}_{2} \mathrm{O} / \mathrm{g}$ para coroa, ambos em base seca (B.S.). Os valores mínimo e máximo obtidos para essa característica foram de 1,13 e 1,26, para casca e 0,857 e 1,790 para coroa. A alta umidade impõe a necessidade de secagem para evitar custos de transporte e obter maior tempo de prateleira, além de agregar valor ao resíduo, por obter um novo produto.

A atividade do resíduo de abacaxi, por sua vez, apresentou valores de 0,914 e 0,921, para casca e coroa, respectivamente, o que fornece evidência adicional da necessidade de realizar a secagem desse resíduo, já que, encontra-se muito acima do limite máximo de atividade de água $(0,6)$ abaixo do qual não ocorre proliferação de microrganismos (Jay, 2005).

Estão representadas na Figura 1as isotermas de dessorção do resíduo (casca e coroa) de abacaxi representadas na forma $X_{\text {eq }}$ em função $a_{w}$ para as temperaturas de $25,30,35$ e 40 ${ }^{\circ} \mathrm{C}$.

Observando-se os dados experimentais obtidos da atividade de água $\left(\mathrm{a}_{\mathrm{w}}\right)$ com suas respectivas umidades de equilíbrio $\left(\mathrm{X}_{\mathrm{eq}}\right)$ (Figura 1) em diferentes temperaturas, verifica-se que as umidades de equilíbrio $\left(\mathrm{X}_{\mathrm{eq}}\right)$ para o resíduo de abacaxi aumentam com o aumento da atividade de água $\left(\mathrm{a}_{\mathrm{w}}\right)$, resultado que concorda com o que ocorre para a maioria dos produtos higroscópicos, tendo sido observado por Oliveira et al. (2009), ao avaliarem isotermas de dessorção da coroa de abacaxi in natura nas mesmas temperaturas estudadas nesse trabalho, assegurando assim nossa investigação, visto que o nosso material seria submetido a fermentação semi-sólida, nas mesmas condições das do referido autor, sendo o nosso utilizado posteriormente em secagem.

Os pontos experimentais das isotermas de dessorção para o resíduo (casca e coroa) de abacaxi estão praticamente alinhados nas quatro temperaturas estudadas, verifica-se então o pouco efeito da temperatura sobre as quatro isotermas, podendo ser justificado pela pequena 
variação nos níveis de temperatura estudada $\left(25\right.$ a $\left.40{ }^{\circ} \mathrm{C}\right)$. Comportamento similar foi encontrado por Oliveira et al. (2006), ao estudarem as isotermas de dessorção da casca do maracujá (Passiflora edulis Sims). Verifica-se que as curvas apresentam comportamento típico de isotermas do tipo III, de acordo com classificação de Brunauer et al. (1938).
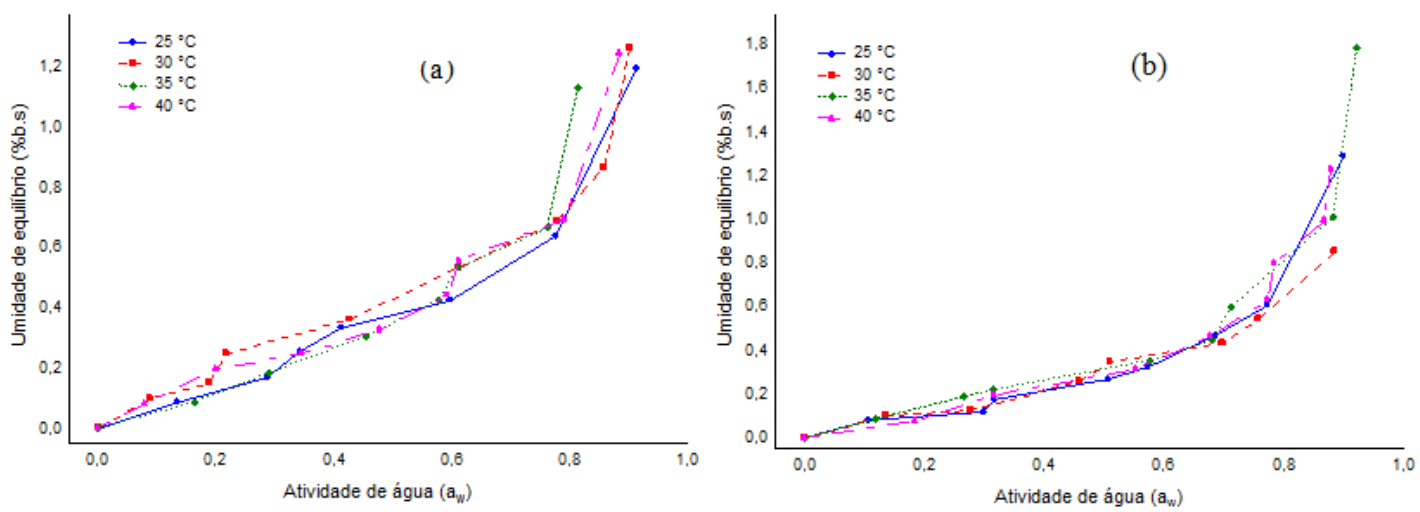

Figura 1. Isotermas de dessorção de umidade da casca (a) e coroa (b) de abacaxi

Conforme os resultados apresentados na Tabela 1 verifica-se que o modelo de GAB representou com mais precisão os dados experimentais para o resíduo (casca) de abacaxi, apresentando $\mathrm{R}^{2}$ acima de $98 \%$ para todas as temperaturas e o menor valor para do desvio percentual médio $(\mathrm{P})$, com exceção da temperatura de $35^{\circ} \mathrm{C}$ que apresentou um valor menor de $\mathrm{P}$ para o modelo de BET linearizado.

Tabela 1. Parâmetros de ajuste dos modelos das isotermas de dessorção do resíduo (casca) de abacaxi, coeficientes de determinação $\left(\mathrm{R}^{2}\right)$ e desvios percentuais médios $(\mathrm{P})$.

\begin{tabular}{lcccccc}
\hline & $\begin{array}{c}\text { Temperatura } \\
\left({ }^{\mathbf{}} \mathbf{C}\right)\end{array}$ & $\mathbf{X}_{\mathbf{m}}$ & $\mathbf{C}$ & $\mathbf{K}$ & $\mathbf{R}^{\mathbf{2}}$ & $\mathbf{P}(\boldsymbol{\%})$ \\
\cline { 2 - 7 } GAB & 25 & 0,2152 & 6,7166 & 0,9019 & 0,9962 & 13,05 \\
& 30 & 0,2146 & 13,9128 & 0,9120 & 0,9906 & 14,71 \\
& 35 & 0,1841 & 6,3718 & 1,0231 & 0,9831 & 13,72 \\
& 40 & 0,2064 & 11,7087 & 0,9406 & 0,9912 & 9,71 \\
\hline \multirow{4}{*}{ BET } & Temperatura & $\mathbf{X}_{\mathbf{m}}$ & & $\mathbf{C}$ & $\mathbf{R}^{\mathbf{2}}$ & $\mathbf{P}(\boldsymbol{\%})$ \\
\cline { 2 - 7 } Linearizado & $\left({ }^{\mathbf{C}} \mathbf{)}\right)$ & 0,1109 & & 13,3569 & 0,9596 & 28,11 \\
& 25 & 0,1289 & & 8,9867 & 0,9684 & 22,81 \\
& 30 & 0,2060 & & 4,4044 & 0,9827 & 11,09 \\
& 35 & 0,1475 & & 8,0289 & 0,9786 & 11,64 \\
\hline
\end{tabular}


De acordo ainda com os resultados da Tabela 1, observa-se que o conteúdo de umidade da camada monomolecular $\left(\mathrm{X}_{\mathrm{m}}\right)$ do modelo de $\mathrm{GAB}$ apresentou valores próximos nas diferentes temperaturas, apresentando um valor médio de 0,2051. Esse valor de $X_{m}$ encontra-se acima do valor determinado para alimentos por Labuza et al. (1985), que indicam como valor máximo para $\mathrm{X}_{\mathrm{m}}$ de $10 \%$ (b.s). Em relação ao parâmetro $\mathbf{C}$ não se observa uma tendência nos seus respectivos valores em relação às temperaturas estudadas. Quanto ao parâmetro K do modelo de GAB, que representa o fator de correlação das propriedades das moléculas na multicamada com relação ao volume do líquido, observam-se valores em torno de um (1). Tem-se o aumento desse parâmetro com o aumento da temperatura, para faixa de temperatura entre $25 \mathrm{a} 35^{\circ} \mathrm{C}$.

Na Tabela 2, encontram-se os parâmetros obtidos por regressão não-linear para os modelos GAB e BET Linearizado para o ajuste das isotermas de dessorção da coroa de abacaxi em cada temperatura estudada, assim como os coeficientes de determinação $\left(\mathrm{R}^{2}\right)$ e desvio percentual médio $(\mathrm{P})$.

Verificou-se que GAB foi o modelo que apresentou um bom ajuste para todas as isotermas estudadas. Este modelo é amplamente utilizado, para descrever o comportamento de isotermas de alimentos por diversos pesquisadores. Telis et al. (2000) obtiveram um bom ajuste de GAB aos dados experimentais de isotermas de casca e polpa de uva, para temperaturas de 20 a $70{ }^{\circ} \mathrm{C}$ e atividades de água entre 0,02 e 0,85 e Waughon \& Pena (2007) obtiveram bons ajustes do modelo $\mathrm{GAB}$ aos dados de dessorção de umidade das fibras residuais do abacaxi, após secagem. Os valores referentes ao conteúdo de umidade da monocamada molecular $\left(\mathrm{X}_{\mathrm{m}}\right)$, do modelo de $\mathrm{GAB}$, flutuaram aleatoriamente dentro da faixa de temperatura estudada (Tabela 2).

Tabela 2. Parâmetros de ajuste dos modelos das isotermas de dessorção do resíduo (coroa) de abacaxi, coeficientes de determinação $\left(\mathrm{R}^{2}\right)$ e desvios percentuais médios $(\mathrm{P})$

\begin{tabular}{lcccccc}
\hline & $\begin{array}{c}\text { Temperatura } \\
\left({ }^{\mathbf{O}} \mathbf{C}\right)\end{array}$ & $\mathbf{X}_{\mathbf{m}}$ & $\mathbf{C}$ & $\mathbf{K}$ & $\mathbf{R}^{\mathbf{2}}$ & $\mathbf{P}(\boldsymbol{\%})$ \\
\cline { 2 - 7 } GAB & 25 & 0,1649 & 4,6616 & 0,9740 & 0,9905 & 8,12 \\
& 30 & 0,2036 & 4,7623 & 0,8755 & 0,9955 & 10,88 \\
& 35 & 0,1382 & 3,8618 & 0,9994 & 0,9908 & 15,38 \\
& 40 & 0,1920 & 3,4945 & 0,9608 & 0,9918 & 9,49 \\
\hline BET & Temperatura & $\mathbf{X}_{\mathbf{m}}$ & & $\mathbf{C}$ & $\mathbf{R}^{\mathbf{2}}$ & $\mathbf{P}(\boldsymbol{\%})$ \\
Linearizado & $\left({ }^{\circ} \mathbf{C}\right)$ & 0,1344 & 13,064 & 0,9968 & 10,80 \\
& 25 & 0,1101 & & 8,5724 & 0,9599 & 8,89 \\
& 30 & 0,1373 & & 40,8746 & 0,9908 & 15,38 \\
& 35 & 0,1493 & & 9,8414 & 0,9892 & 12,32 \\
\hline
\end{tabular}

O valor de $X_{m}$ tem especial importância uma vez que, conforme Mishra et al. (1996), a umidade na monocamada determina o teor de umidade para uma armazenagem segura, na qual o material tem estabilidade máxima. Em relação à constante de sorção $\mathrm{C}$ do modelo de $\mathrm{GAB}$, que é função das interações entre os sítios ativos do produto e as moléculas de água, 
observa-se que os valores são próximos para as temperaturas de 25 e $30^{\circ} \mathrm{C}$, o mesmo acontecendo para as temperaturas 35 e $40{ }^{\circ} \mathrm{C}$. O valor de $\mathrm{K}$ ficou praticamente constante, isto é, em torno de 1 com exceção da temperatura de $30{ }^{\circ} \mathrm{C}$.

Dessa forma, utilizou-se neste trabalho a equação de GAB para ajustar as isotermas de dessorção do resíduo (casca e coroa). A Figura 2 apresenta as isotermas de dessorção do resíduo de abacaxi (casca e coroa) nas temperaturas de $25,30,35$ e $40{ }^{\circ} \mathrm{C}$, ajustadas pelo modelo de GAB.
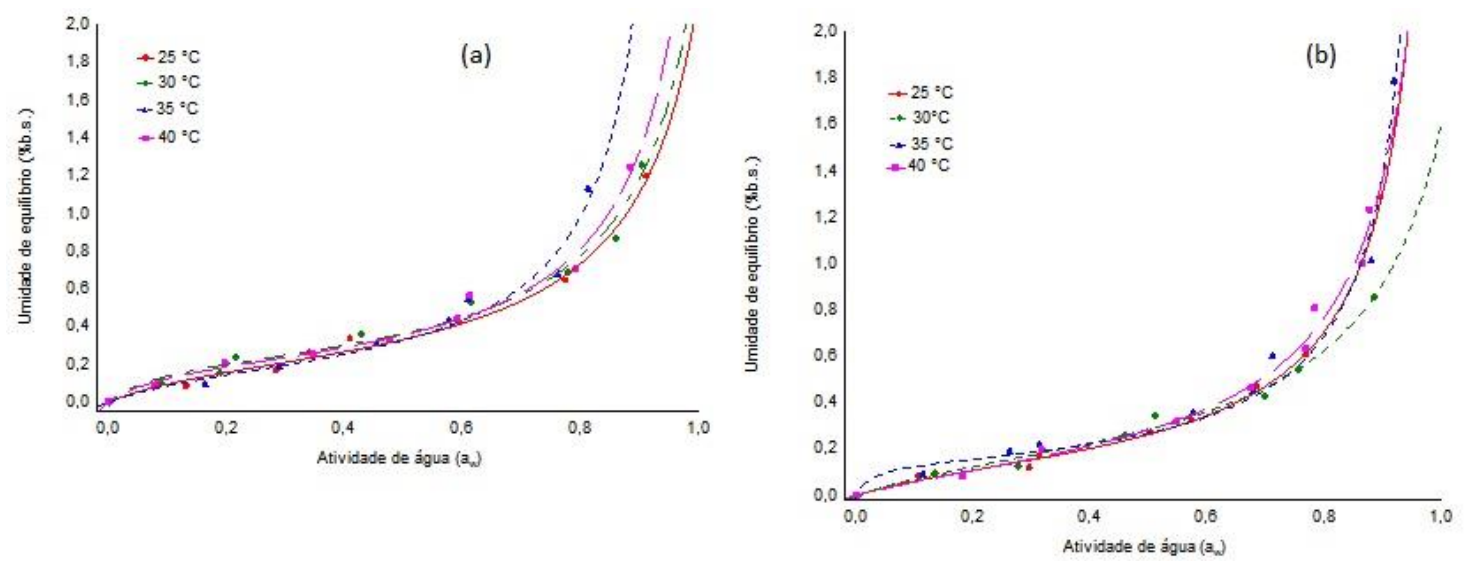

Figura 2. Isotermas de dessorção de umidade de abacaxi: casca (a) e coroa (b), ajuste pelo modelo de GAB

\section{CONCLUSÕES}

O resíduo de abacaxi (casca e coroa) apresenta elevado teor de umidade e atividades de água, necessitando de uma etapa de secagem previamente ao seu armazenamento. O modelo de GAB foi o mais adequado para descrever isotermas de dessorção de umidade em um intervalo de atividade de água entre 0,079 e 0,914 para casca e 0,106 e 0,921 para coroa, nas temperaturas de $25,30,35$ e $40^{\circ} \mathrm{C}$.

\section{REFERÊNCIAS}

AKANBI, C.T.; ADEYEMI, R.S.; OJO, A. Drying characteristics and sorption isotherm of tomato slices. J. Food Eng., v.73, n.2, p.157-163, 2006.

ALEXANDRE, H. V.; FIGUEIRÊDO, R. M. F. DE; QUEIROZ, A. J. DE M. Isotermas de adsorção de umidade da pitanga em pó. Rev. Biol. Ciênc. Ter., v.7, p.11-20, 2007.

ASSUNÇÃO, A.B.; PENA, R.S. Comportamento higroscópico do resíduo seco de camarão rosa. Ciênc. Tecnol. Alimen., v.27, n.4, p.786-793, 2007.

BRASIL. Instituto Adolfo Lutz. Normas analíticas do instituto Adolfo Lutz - Métodos químicos e físicos para análise de alimentos. 2.ed. São Paulo,v.1, 2005. 
BRITO NETO, J. F.; PEREIRA, W. E; SÁ SOBRINHO, R. G.; BARBOSA; J. A.; SANTOS, D. P. Aspectos produtivos da abacaxicultura familiar e comercial no estado da Paraíba. Rev. Caat., v.21, p.43-50, 2008.

BRUNAUER, S.; EMMET, T.H.; TELLER, F. Adsorption of gases in multimolecular layers. J. Am. Chem. Soc., v.60, p.309-319, 1938.

FURMANIAK, S.; TERZYK, A.P.; GAUDEN, P.A.; RYCHLICKI, G. Applicability of the generalised D'Arcyand Watt model to description of water sorption on pineapple and other food stuffs. J. FoodEng., v.79, n.2, p.718-723, 2007.

HELDMAN, D.R.; HARTEL, R.W. Principles of food processing. New York: Chapman \& Hall, 218p, 2000.

IBGE - Instituto Brasileiro de Geografia e Estatística. Banco de dados agregados. Disponível em: http://www.sidra.ibge.gov.br. Acesso em: 25 jul. 2010.

JAY, J. M. Microbiologia de alimentos. 6 ed. - Porto Alegre: Artmed, 2005.

LABUZA, T.P.; KAANANE, A.; CHEN, J.Y. Effects of temperature on the moisture sorption isotherms and water activity shift of two dehydrated foods. J. Food Sci., v.50, p.385-389, 1985.

MISHRA, V. K.; ORAIKUL, B.; TEMELLI, F. Physical characterization and water sorption of freeze dried dulse Palmaria palmate powder. J. Food Proc. Pres.,Westport, v.20, p.25- 39, 1996.

OLIVEIRA, M. M.; CAMPOS, A. R. N.; DANTAS, J. P.; GOMES, J. P.; SILVA, F. L. H.

Isotermas de dessorção da casca do maracujá (Passiflora edulis Sims): determinação experimental e avaliação de modelos matemáticos. Ciênc. Rur., v. 36, n. 5, 2006.

OLIVEIRA, M.M.; CAMPOS, A.R.N.; SILVA, F.L.H. da.; GOMES, J.P. Isotermas de dessorção da coroa do abacaxi. Tec. Ciênc. Agropec., v.3, p.47-52, 2009.

PELIZER, L. H.; PONTIERI, M. H.; MORAES, I. O. Utilização de resíduos agroindustriais em processos biotecnológicos como perspectiva de redução do impacto ambiental. J. Technol. Manag. Innov., v.2, p.118-127, 2007.

SOUSA, K. A.; RESENDE, O.; COSTA, L. M. Isotermas de dessorção das sementes de nabo forrageiro obtidas pelos métodos dinâmico e estático. Rev. Bras. Eng. Agr. Amb., v.17, p.216222, 2013.

TELIS, V.R.N.; GABAS, A.L.; MENEGALLI, F.C.; TELIS, R.J. Water sorption thermodynamic properties applied to persimmon skin and pulp. Therm. Acta., v.343, p.49-56, 2000 .

WAUGHON, T.G.; PENA, R.S. Avaliação higroscópica da fibra residual do abacaxi. Rev. Bras. Tecnol. Agro., v.1, p.83-92, 2007. 